\title{
LISTA ACTUALIZADA Y BIBLIOGRAFÍA DE LOS CUMÁCEOS (PERACARIDA: CUMACEA) DEL GOLFO Y CARIBE MEXICANOS
}

\section{Updated list and bibliography of Cumacea (Peracarida: Cumacea) of the Caribbean and Gulf of Mexico}

\begin{abstract}
Angeles Jaqueline Ramírez-Villalobos, ${ }^{1, *}, 2$, Manuel Ortiz ${ }^{3}$ y Mario Modesto Chávez Arteaga ${ }^{4}$
'Posgrado en Ciencias Biológicas, Unidad de Posgrado, Edificio A, $1^{\circ}$ Piso, Circuito de Posgrados, Ciudad Universitaria, Coyoacán, C.P. 04510, Distrito Federal, México. ${ }^{2}$ Laboratorio de Zoología. Facultad de Estudios Superiores Iztacala, UNAM, Av. de los Barrios \#1, Col. Los Reyes Ixtacala, Tlalnepantla, Estado de México, C. P. 54090. México; (1) orcid.org/0000-0002-2277-183X. ${ }^{3}$ Laboratorio de Carcinología. Facultad de Estudios Superiores Iztacala, UNAM, Av. de los Barrios \#1, Col. Los Reyes Ixtacala, Tlalnepantla, Estado de México, C. P. 54090. México; (1) orcid.org/0000-0002-6985-8019. ${ }^{4}$ Laboratorio de Limnología. Facultad de Estudios Superiores Iztacala, UNAM, Av. de los Barrios \#1, Col. Los Reyes Ixtacala, Tlalnepantla, Estado de México, C. P. 54090. México; (1) orcid.org/0000-0001-6185-9909. *Para correspondencia: jaky@comunidad.unam.mx.
\end{abstract}

\section{RESUMEN}

Se realizó la compilación bibliográfica (hasta agosto de 2019), de las especies de cumáceos reportados en el Golfo de México y Mar Caribe. Un total de 25 especies, nueve géneros y cinco familias son enlistadas. Se tiene registro de cumáceos en cuatro de los seis estados cuyo litoral se encuentra en el Golfo de México y o mar Caribe, en Veracruz se han reportado seis especies en siete localidades, en los límites de este estado con Tabasco dos especies, para Tabasco una especie en una localidad, en Campeche, en tres localidades, tres especies y para Quintana Roo 11 especies en dos localidades. Se tienen más de 30 años sin determinar hasta nivel específico los organismos recolectados, lo que muestra la necesidad de un mayor trabajo taxonómico en este grupo.

Palabra clave: cumáceos, recopilación bibliográfica, aguas mexicanas; crustáceos marinos.

\section{ABSTRACT}

A bibliographic compilation was made (up to August 2019), on cumacean species reported in Mexico Gulf of Mexico and the Caribbean Sea. The total list includes 25 species, nine genera and five families. Cumaceans have been recorded in 4 of the 6 states whose coastline is on the Gulf of Mexico and Caribbean Sea. In Veracruz six species have been reported in seven locations; two species in Veracuz's limits with Tabasco; in one location of Tabasco, one species; in three locations of Campeche, three species and eleven species in two locations of Quintana Roo. For more than 30 years, the collected organisms have not been determined to an specific level, this shows the necessity for more taxonomic work in this group.

Keywords: cumaceans, bibliographic compilation, Mexican waters, marine crustaceans.

\section{INTRODUCCIÓN}

Aunque dentro de los peracáridos los mejor estudiados son los anfípodos y los isópodos, los cumáceos comenzaron a ser mayormente estudiados debido a varias causas como lo son: el descubrimiento de formas fósiles; su importancia para descubrir las relaciones filogenéticas tanto dentro del grupo, como con los demás miembros de la clase Malacostraca (Haye, Kornfield y Watling, 2004; Spears et al. 2005) y el ser importantes en la dieta de peces carnívoros demersales tanto en ambientes naturales como en cultivados (Băcescu y Petrescu 1999). 
El primer trabajo que reporta cumáceos para el Golfo de México y Mar Caribe (en territorio mexicano), es el de Băcescu, 1971, donde reporta la nueva especie Cumella (Cumella) meredithi fuera del arrecife Isla de Media, Veracruz (Gulf of Mexico, Veracruz, $100 \mathrm{ft}$. off the reef of Isla de Media, tomado del original).

Diez años después del primer trabajo para la zona, Rhada Devi y Kurian(1981), describen dos nuevas especies: Pseudocyclaspis mexicansis (actualmente válida como Cyclaspis mexicansis) y Oxyurostila atlantica, cuya localidad en México corresponde al estado de Campeche.

Donath-Hernández (1988a) amplía la distribución de Cyclaspis bacescui, C. dentifrons, C. longipes, C. oxyura, C. unicornis, C. varians, Spilocuma salomani, Vaunthompsonia floridana, V. minor, Eudorella monodon, Campylaspis heardi, Cumella (Cumella) clavicauda, C. (C.) garrityi, C. (C.) sp. aff. garrityi, C. (C.) vicina, al Golfo de México y/o mar Caribe.

Markham, et al. (1990) publican un trabajo sobre los crustáceos marinos de la costa caribeña de Quintana Roo, México, información obtenida de las colectas realizadas en distintas localidades o de trabajos anteriores. En este trabajo se reportan 11 especies de cumáceos, previamente reportadas por Donath-Hernández (1988a) y se reportan cinco especies nuevas que no fueron determinadas y sólo se presentan como sigue: Cyclaspis n. sp., Campylaspis n. sp. Cumella (Cumewingia) n. sp., Cumella n. sp. y Oxiurostylis sp. (n. sp.?).

En 1992, Donath-Hernández reporta una nueva especie, Cumella (Cumewingia) siankaana, de Bahía de la Ascensión, Quintana Roo. Para el 2002 se encuentran los trabajos de Cházaro-Olvera et al., donde reportan una especie de cumáceo, Cyclaspis sp., y también el de García-Madrigal et. al., quienes publican las especies de crustáceos presentes en la Colección de Referencia de Bentos Costero de ECOSUR, reportando 17 especies de cumáceos. En 2004, Roccatagliata elaboró un resumen de las especies de cumáceos reportadas, hasta ese momento, para México. Él menciona un total de 32 especies para el país, 13 especies reportadas para el Pacífico y 19 para el Golfo de México y mar Caribe, de estas últimas, 11 especies son las localizadas en el mar Caribe, con sólo una endémica de México (Cumella siankaana); las ocho restantes se localizan en el Golfo de México, cinco de ellas compartidas con Estados Unidos y tres exclusivas del territorio mexicano (Cyclaspis mexicansis, Cumella meredithi y Oxyurostylis atlantica).

En 2009 se publica el libro Gulf of Mexico-Origins, Waters and Biota, en él se encuentra el capítulo de cumáceos de Heard y Roccatagliata, donde reportan un total de 40 especies, en este trabajo se incluyen especies que están tanto en territorio mexicano como estadounidense.

En 2011, Winfield y Ortiz forman parte de la publicación La Biodiversidad en Veracruz, estudio de estado. Vol. III, aportando el capítulo Crustáceos con bolsa incubadora (Crustacea: Malacostraca: Peracarida), en el se mencionan cuatro especies de cumáceos que se han reportado para el estado.

En el trabajo Crustáceos del Sistema Arrecifal Veracruzano de Hermoso-Salazar y Arvizu-Coyotzi (2015) mencionan a la especie Cumella meredithi, por ser un cumáceo encontrado en Veracruz.

Los listados actualizados de especies son de suma importancia, ya sea para incrementar el conocimiento de la diversidad de alguna región, para proporcionar cifras y datos que puedan ser comparados con otras partes del mundo, para definir zonas prioritarias de conservación, para servir como punto de partida en los estudios sobre redes tróficas, etc. (May, 1992; Hendrickx, 1995; Ortiz et al., 2001). En este caso en particular nos proporciona información sobre el conocimiento 
que se tiene de los cumáceos y los lugares en México, donde ha sido estudiados mayoritariamente, presentando una visión de posibles lugares de investigación que incrementarían el conocimiento del grupo.

\section{OBJETIVO}

- Presentar una lista actualizada de las especies de cumáceos mexicanos.

\section{MATERIALES Y MÉTODOS}

Este trabajo se basa en la compilación de literatura: artículos, libros y capítulos de libros (no se consideraron tesis ni reportes de proyectos), de los cumáceos reportados para México. Se incluye la localidad, las coordenadas geográficas y/o la profundidad en que fueron encontrados (estos datos se tomaron de las publicaciones en las que están reportados los datos). En los casos en donde sólo se reportaron las coordenadas sin especificar localidad, esta se determinó mediante el uso de Google Earth 2020.

Para el arreglo taxonómico se siguió el propuesto por Ahyong et al. (2011), para nivel supragenérico, mientras que para los niveles de género y especie el arreglo fue alfabético. La nomenclatura de las especies fue actualizada con base en Watling y Gerken (2019) y en WoRMS Editorial Board (2019).

\section{RESULTADOS Y DISCUSIÓN}

Se enlista un total de 25 especies, nueve géneros y cinco familias, además se incluyen cinco especies señaladas como especies nuevas (o posibles), por los autores que las reportan (Cyclaspis $n$. $s p$, Campylaspis n. sp., Oxyurostylis sp. [n. sp.?], Cumella n. sp. y Cumella Cumewingia n. sp.) y una especie que no fue determinada (Cyclaspis $s p$.).

Los cumáceos han sido reportados en cuatro de los seis estados que conforman el litoral del Golfo de México y/o mar Caribe. En Veracruz se han reportado seis especies en siete localidades (Băcescu, 1971; Donath-Hernández, 1988a); en los límites de Veracruz y Tabasco dos especies (Donath-Hernández, 1988a); en el estado de Tabasco hay registro de una especie en una localidad (Donath-Hernández, 1988a); para Campeche se reportan, tres especies en tres localidades (Rhada Devi y Kurian, 1981; Donath-Hernández, 1988a) y, finalmente, para Quintana Roo hay reporte de 10 especies en una localidad (Bahía de la Ascensión ) y una especie en otra (Playa Aventuras), (Donath-Hernández, 1988a, 1992).

Como parte de la revisión bibliográfica se encontró que una nueva especie nombrada por Petrescu en 2018 (registrada para Australia), entra en homonimia con la especie Campylaspis heardi de Muradian-Ciamician, 1980, situación que se le notificó a Petrescu.

Se hace notar la imprecisión con la localidad de Cumella (Cumella) meredithi Băcescu, 1971, por la ausencia de coordenadas y cambio de nombre en la localidad, señaladas en las publicaciones del autor (Băcescu, 1971, 1992) y en el trabajo de recopilación de Roccatagliata (2004).

Es importante notar que el trabajo que presenta mayor número de especies reportadas para el área es el de Donath-Hernández, 1988a, donde se reportan 15 especies, además es el último trabajo donde se ha colectado un organismo determinado hasta nivel de especie (Eudorella monodon colectada en agosto de 1987 en las localidades de Alvarado y Cercano a los Tuxtlas, Veracruz). En cuanto al trabajo de García-Madrigal et al. (2002), donde reportan 17 especies depositadas 
en la Colección de Referencia de Bentos Costero de ECOSUR, no se señalan las localidades, e incluso cuatro de las especies reportadas han sido reportadas para el Pacífico mexicano Cyclaspis bituberculata reportada por Donath-Hernández (1988b), C. nubila reportada por Donath-Hernández (1987a,b, 1988b, 1993), Leptocuma forsmani reportada por Zimmer (1943), Dexter (1976), Donath-Hernández (1987b) y Jarquín (2013), y por último Hemilamprops californicus reportada por Donath-Hernández (1987b).

Tabla 1. Lista sistemática de las especies de cumáceos reportadas para el Golfo de México y Mar Caribe

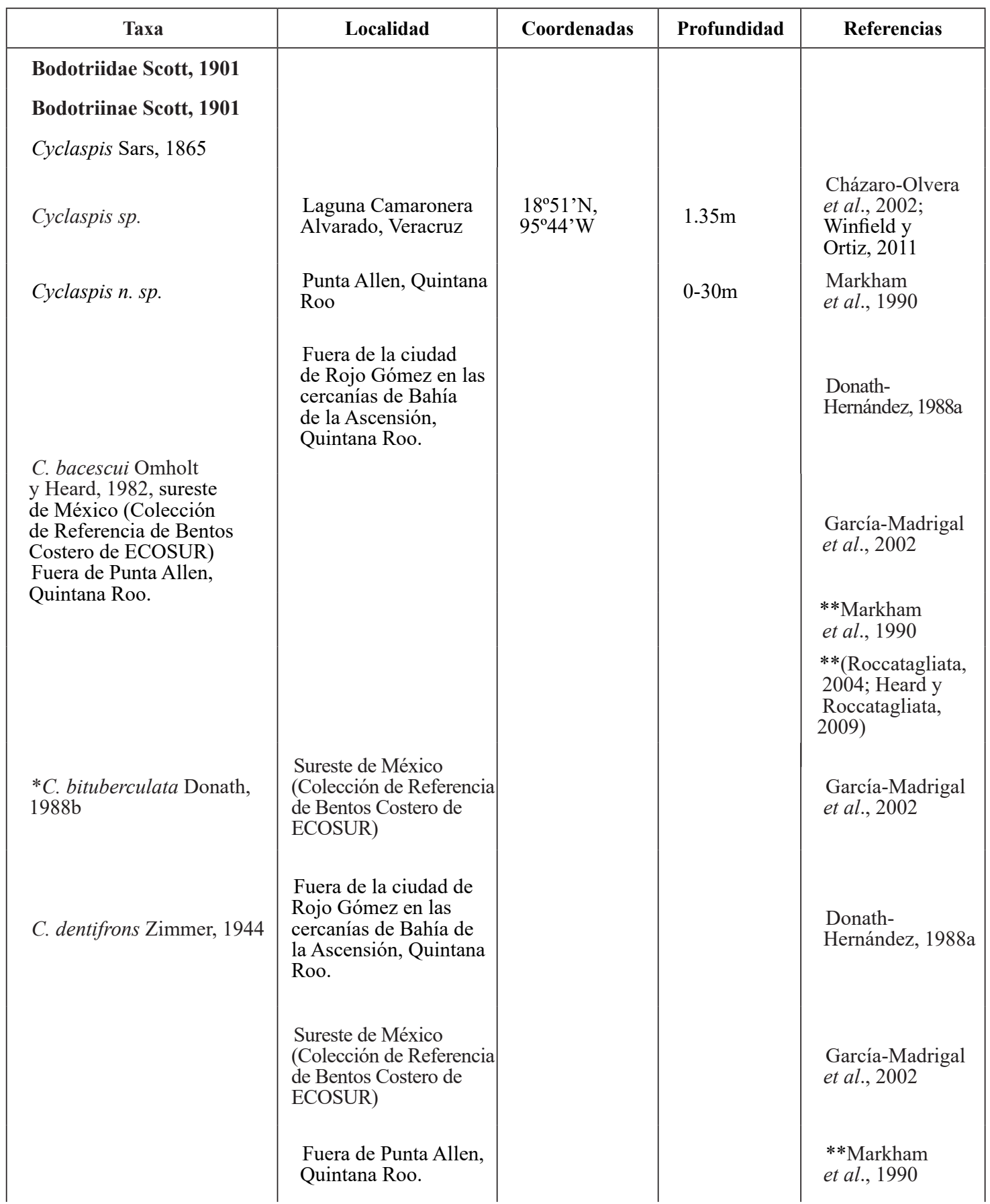




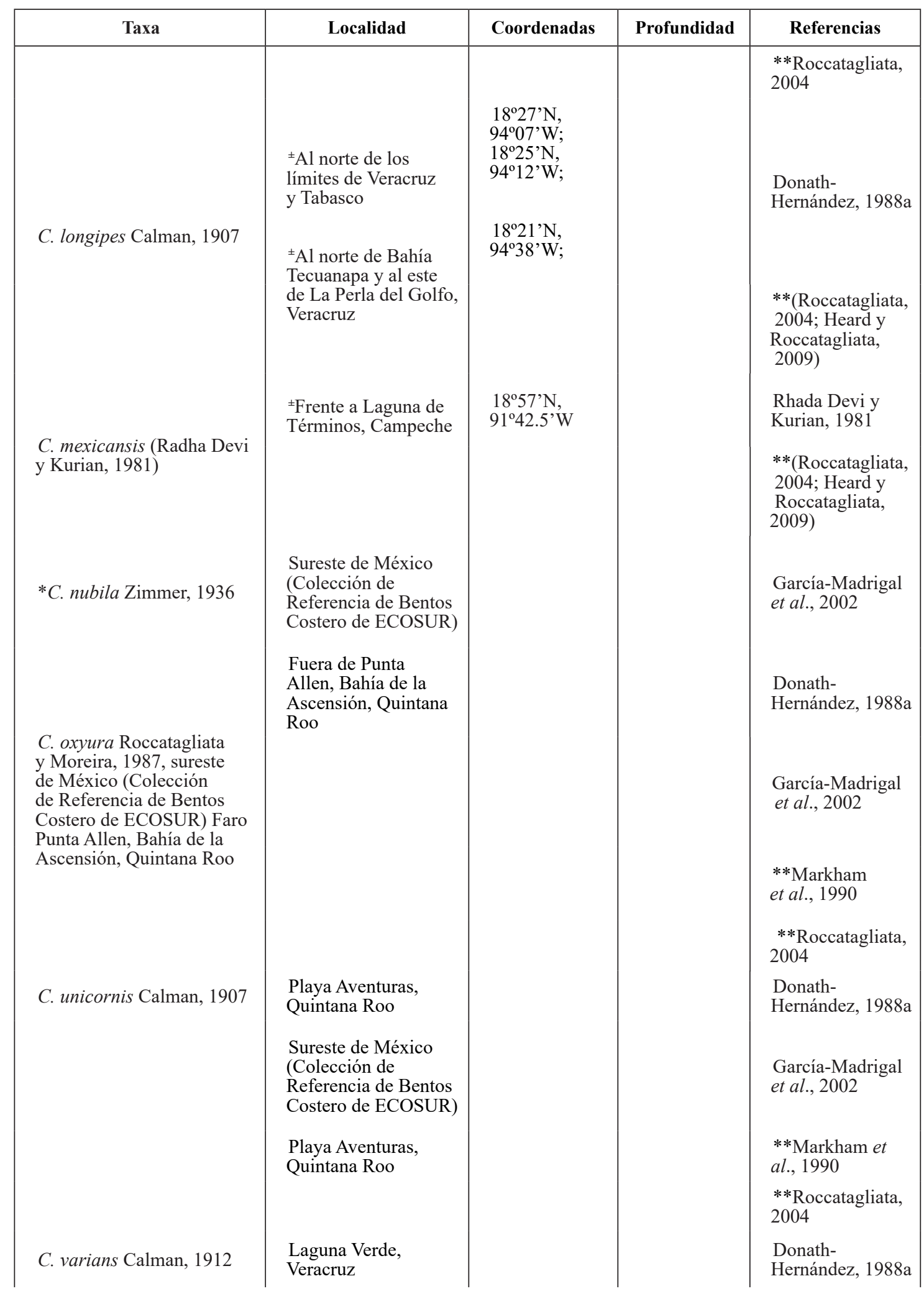


Tabla I. Continuación

\begin{tabular}{|c|c|c|c|c|}
\hline Taxa & Localidad & Coordenadas & Profundidad & Referencias \\
\hline & $\begin{array}{l}\text { Sureste de México } \\
\text { (Colección de } \\
\text { Referencia de Bentos } \\
\text { Costero de ECOSUR) }\end{array}$ & & & $\begin{array}{l}\text { García-Madrigal } \\
\text { et al., } 2002 \\
\text { **(Roccatagliata, } \\
\text { 2004; Heard y } \\
\text { Roccatagliata, } \\
\text { 2009; Winfield } \\
\text { y Ortiz, 2011) }\end{array}$ \\
\hline \multicolumn{5}{|l|}{$\begin{array}{l}\text { Mancocumatinae Watling, } \\
1977\end{array}$} \\
\hline \multicolumn{5}{|l|}{ Spilocuma Watling, 1977} \\
\hline S. salomani Watling, 1977 & $\begin{array}{l}\text { Playa } \\
\text { Chalchiuhcueyehcan, } \\
\text { ejido de la Antigua, } \\
\text { Veracruz }\end{array}$ & & & $\begin{array}{l}\text { Donath- } \\
\text { Hernández, 1988a }\end{array}$ \\
\hline & & & & $\begin{array}{l}* *(\text { Roccatagliata, } \\
\text { 2004; Heard y } \\
\text { Roccatagliata, } \\
\text { 2009; Winfield y } \\
\text { Ortiz, 2011) }\end{array}$ \\
\hline \multicolumn{5}{|l|}{$\begin{array}{l}\text { Vaunthompsoniinae } \\
\text { G. O. Sars, } 1878\end{array}$} \\
\hline \multicolumn{5}{|l|}{ Leptocuma Sars, 1873} \\
\hline *L. forsmani Zimmer, 1943 & $\begin{array}{l}\text { Sureste de México } \\
\text { (Colección de } \\
\text { Referencia de Bentos } \\
\text { Costero de ECOSUR) }\end{array}$ & & & $\begin{array}{l}\text { García-Madrigal } \\
\text { et al., } 2002\end{array}$ \\
\hline \multicolumn{5}{|l|}{ Vaunthompsonia Bate, 1858} \\
\hline V. floridana Băcescu, 1971 & $\begin{array}{l}\text { Fuera de la ciudad de } \\
\text { Rojo Gómez en las } \\
\text { cercanías de Bahía } \\
\text { de la Ascensión, } \\
\text { Quintana Roo. }\end{array}$ & & & $\begin{array}{l}\text { Donath- } \\
\text { Hernández, 1988a }\end{array}$ \\
\hline & $\begin{array}{l}\text { Sureste de México } \\
\text { (Colección de } \\
\text { Referencia de Bentos } \\
\text { Costero de ECOSUR) }\end{array}$ & & & $\begin{array}{l}\text { García-Madrigal } \\
\text { et al., } 2002\end{array}$ \\
\hline & $\begin{array}{l}\text { Fuera de Punta Allen } \\
\text { y Cayo Valencia, } \\
\text { Quintana Roo }\end{array}$ & & & $\begin{array}{l}\text { **Markham } \\
\text { et al., } 1990 \\
* * \text { Roccatagliata, } \\
2004\end{array}$ \\
\hline $\begin{array}{l}\text { V. minor Zimmer, } 1944 \\
\text { (=Vaunthompsonia cf. } \\
\text { cristata Bate, } 1858 \text { fide } \\
\text { Petrescu, 2002) }\end{array}$ & $\begin{array}{l}\text { Fuera de Punta } \\
\text { Allen, Bahía de la } \\
\text { Ascensión, Quintana } \\
\text { Roo }\end{array}$ & & & $\begin{array}{l}\text { Donath- } \\
\text { Hernández, 1988a }\end{array}$ \\
\hline & $\begin{array}{l}\text { Sureste de México } \\
\text { (Colección de } \\
\text { Referencia de Bentos } \\
\text { Costero de ECOSUR) }\end{array}$ & & & $\begin{array}{l}\text { García-Madrigal } \\
\text { et al., } 2002\end{array}$ \\
\hline
\end{tabular}




\begin{tabular}{|c|c|c|c|c|}
\hline Taxa & Localidad & Coordenadas & Profundidad & Referencias \\
\hline & $\begin{array}{l}\text { Fuera de Punta Allen, } \\
\text { La Caleta, Bahía de la } \\
\text { Ascensión, Quintana } \\
\text { Roo }\end{array}$ & & & $\begin{array}{l}\text { **Markham } \\
\text { et al., } 1990 \\
\text { **Roccatagliata, } \\
2004\end{array}$ \\
\hline \multicolumn{5}{|l|}{ Diastylidae Bate, 1856} \\
\hline \multicolumn{5}{|l|}{ Oxyurostylis Calman, 1912} \\
\hline Oxyurostylis sp. [n. sp.?] & $\begin{array}{l}\text { La Caleta y Bahía } \\
\text { de la Ascensión, } \\
\text { Quintana Roo }\end{array}$ & & & $\begin{array}{l}\text { Markham et al., } \\
1990\end{array}$ \\
\hline $\begin{array}{l}\text { O. atlantica Rhada Devi y } \\
\text { Kurian, } 1981\end{array}$ & $\begin{array}{l}{ }^{ \pm} \text {Al Noroeste de } \\
\text { Sabancuy y al } \\
\text { Noreste de Laguna de } \\
\text { Términos, Campeche }\end{array}$ & $\begin{array}{l}19^{\circ} 06.8^{\prime} \mathrm{N} \\
91^{\circ} 16.2^{\prime} \mathrm{W}\end{array}$ & & $\begin{array}{l}\text { Rhada Devi y } \\
\text { Kurian, } 1981\end{array}$ \\
\hline & & & & $\begin{array}{l}* *(\text { Roccatagliata, } \\
\text { 2004; Heard y } \\
\text { Roccatagliata, } \\
\text { 2009) }\end{array}$ \\
\hline O. smithi Calman, 1912 & $\begin{array}{l}\text { Sureste de México } \\
\text { (Colección de } \\
\text { Referencia de Bentos } \\
\text { Costero de ECOSUR) }\end{array}$ & & & $\begin{array}{l}\text { García-Madrigal } \\
\text { et al., } 2002\end{array}$ \\
\hline \multicolumn{5}{|l|}{ Lampropidae Sars, 1878} \\
\hline Hemilamprops Sars, 1883 & & & & \\
\hline $\begin{array}{l}\text { *H. californicus Zimmer, } \\
1936\end{array}$ & $\begin{array}{l}\text { Sureste de México } \\
\text { (Colección de } \\
\text { Referencia de Bentos } \\
\text { Costero de ECOSUR) }\end{array}$ & & & $\begin{array}{l}\text { García-Madrigal } \\
\text { et al., } 2002\end{array}$ \\
\hline \multicolumn{5}{|l|}{ Leuconidae Sars, 1878} \\
\hline \multicolumn{5}{|l|}{ Eudorella Norman, 1867} \\
\hline E. monodon Calman, 1912 & $\begin{array}{l}{ }^{ \pm} \text {Alvarado, Veracruz; } \\
{ }^{ \pm} \text {Cercano a los } \\
\text { Tuxtlas, Veracruz } \\
{ }^{ \pm} \text {Al norte de Oyapan } \\
\text { y al nor-noroeste de } \\
\text { la Perla del Golfo, } \\
\text { Veracruz }\end{array}$ & $\begin{array}{l}18^{\circ} 35^{\prime}, \mathrm{N} \\
94^{\circ} 51^{\prime} \mathrm{W} \\
18^{\circ} 32^{\prime}, \mathrm{N} \\
94^{\circ} 38^{\prime} \mathrm{W} \\
\\
18^{\circ} 51^{\prime} \mathrm{N} \\
95^{\circ} 41^{\prime} \mathrm{W}\end{array}$ & & $\begin{array}{l}\text { Donath- } \\
\text { Hernández, 1988a }\end{array}$ \\
\hline & $\begin{array}{l}\text { Al oeste de } \\
\text { Champotón, } \\
\text { Campeche }\end{array}$ & $\begin{array}{l}19^{\circ} 23^{\prime} \mathrm{N} \\
91^{\circ} 19^{\prime} \mathrm{W}\end{array}$ & & \\
\hline & & & & $\begin{array}{l}* *(\text { Roccatagliata, } \\
\text { 2004; Heard y } \\
\text { Roccatagliata, } \\
2009)\end{array}$ \\
\hline Nannastacidae Bate, 1866 & & & & \\
\hline Campylaspis Sars, 1865 & & & & \\
\hline Campylaspis n. sp. & $\begin{array}{l}\text { Punta Allen, } \\
\text { Quintana Roo }\end{array}$ & & & $\begin{array}{l}\text { Markham et al., } \\
1990\end{array}$ \\
\hline
\end{tabular}


Tabla I. Continuación

\begin{tabular}{|c|c|c|c|c|}
\hline Taxa & Localidad & Coordenadas & Profundidad & Referencias \\
\hline $\begin{array}{l}\text { C. heardi Muradian- } \\
\text { Ciamician, } 1980 \text { non } \\
\text { Campylaspis heardi } \\
\text { Petrescu, } 2018\end{array}$ & $\begin{array}{l}{ }^{ \pm} \text {Al norte de } \\
\text { Coatzacoalcos, } \\
\text { Veracruz } \\
{ }^{ \pm} \text {Al norte de los } \\
\text { límites de Veracruz y } \\
\text { Tabasco } \\
\\
{ }^{ \pm} \text {Al norte de Villa } \\
\text { Sánches Magallanes, } \\
\text { Tabasco } \\
\\
{ }^{ \pm} \text {Fuera de la ciudad } \\
\text { de Rojo Gómez, } \\
\text { Fuera de Punta Allen, } \\
\text { Quintana Roo } \\
\text { Sureste de México } \\
\text { (Colección de } \\
\text { Referencia de Bentos } \\
\text { Costero de ECOSUR) } \\
\text { Fuera de Punta Allen, } \\
\text { NE del Golfo de } \\
\text { México, Veracruz }, \\
\text { Quintana Roo }\end{array}$ & $\begin{array}{l}18^{\circ} 21^{\prime} \mathrm{N}, \\
94^{\circ} 25^{\prime} \mathrm{W} ; \\
18^{\circ} 41^{\prime} \mathrm{N}, \\
94^{\circ} 12^{\prime} \mathrm{W} ; \\
18^{\circ} 25^{\prime} \mathrm{N}, \\
94^{\circ} 12^{\prime} \mathrm{W} ; \\
18^{\circ} 27^{\prime} \mathrm{N} \\
94^{\circ} 07^{\prime} \mathrm{W} ; \\
\\
18^{\circ} 32^{\prime} \mathrm{N}, \\
93^{\circ} 48^{\prime} \mathrm{W}\end{array}$ & & $\begin{array}{l}\text { García-Madrigal } \\
\text { et al., } 2002 \\
\text { **Markham } \\
\text { et al., } 1990 \\
\text { **(Roccatagliata, } \\
\text { 2004; Heard y } \\
\text { Roccatagliata, } \\
\text { 2009) }\end{array}$ \\
\hline $\begin{array}{l}\text { Cumella Sars, } 1865 \\
\text { Cumella n. sp. }\end{array}$ & $\begin{array}{l}\text { La Caleta, Bahía de la } \\
\text { Ascensión, Quintana } \\
\text { Roo }\end{array}$ & & & $\begin{array}{l}\text { Markham } \\
\text { et al., } 1990\end{array}$ \\
\hline $\begin{array}{l}\text { Cumella (Cumella) } \\
\text { Sars, } 1865 \\
\text { C.(C.) clavicauda } \\
\text { Calman, } 1911\end{array}$ & $\begin{array}{l}\text { Fuera de la ciudad de } \\
\text { Rojo Gómez en las } \\
\text { cercanías de Bahía } \\
\text { de la Ascensión } \\
\text { y en Bahía de la } \\
\text { Ascensión, } \\
\text { Quintana Roo }\end{array}$ & & & $\begin{array}{l}\text { Donath- } \\
\text { Hernández, 1988a }\end{array}$ \\
\hline & $\begin{array}{l}\text { Sureste de México } \\
\text { (Colección de } \\
\text { Referencia de Bentos } \\
\text { Costero de ECOSUR) } \\
\text { Fuera de Punta Allen, } \\
\text { Quintana Roo. }\end{array}$ & & & $\begin{array}{l}\text { García-Madrigal } \\
\text { et al., } 2002 \\
\text { **Markham et } \\
\text { al., } 1990 \\
\text { **Roccatagliata, } \\
2004\end{array}$ \\
\hline $\begin{array}{l}\text { C. (C.) garrityi Băcescu } \\
\text { y Muradian, } 1977\end{array}$ & $\begin{array}{l}\text { Bahía de la } \\
\text { Ascensión, Quintana } \\
\text { Roo }\end{array}$ & & & $\begin{array}{l}\text { Donath- } \\
\text { Hernández, 1988a }\end{array}$ \\
\hline
\end{tabular}




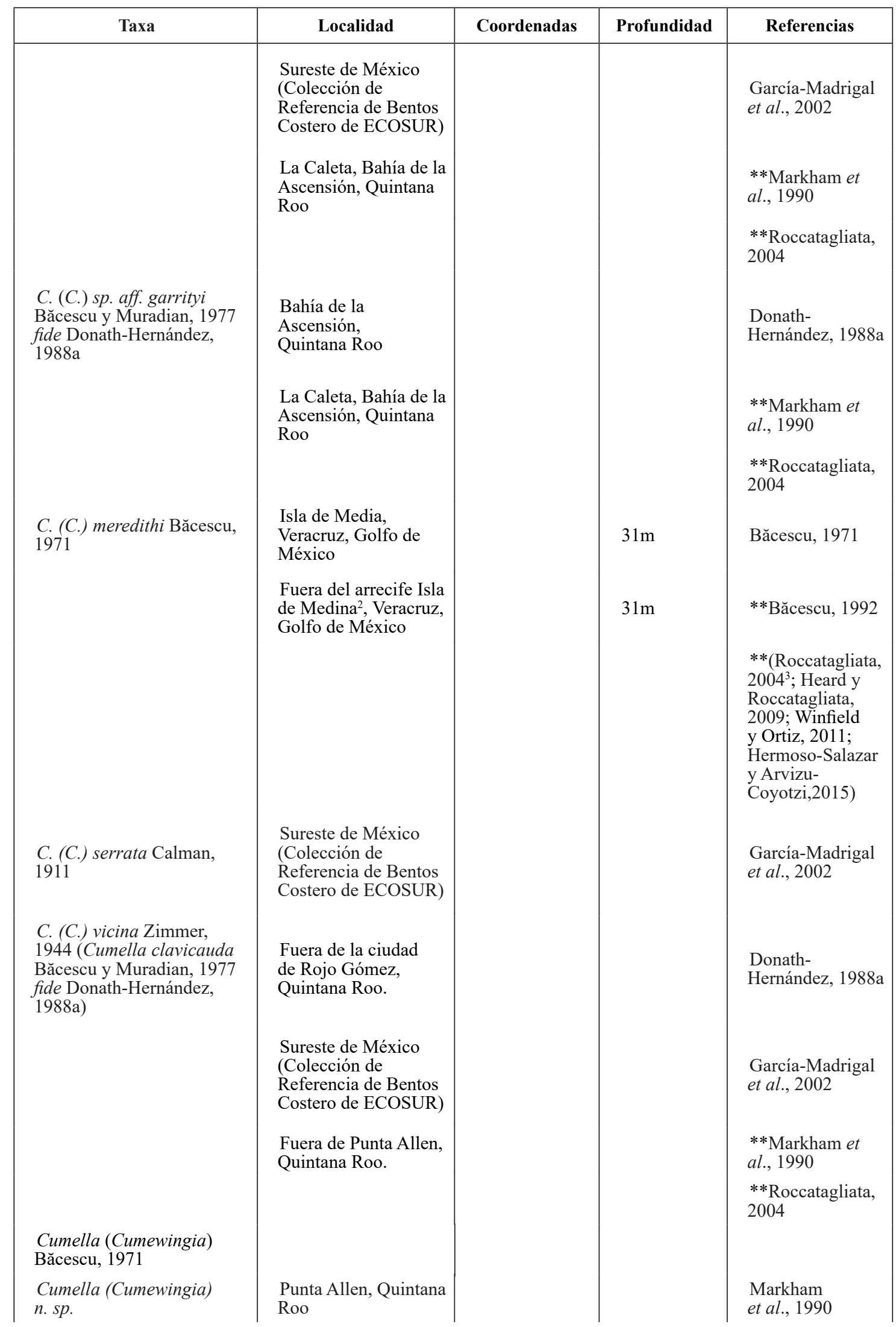


Tabla I. Continuación

\begin{tabular}{|c|c|c|c|c|}
\hline Taxa & Localidad & Coordenadas & Profundidad & Referencias \\
\hline $\begin{array}{l}\text { C. (C.) siankaana Donath- } \\
\text { Hernández, } 1992\end{array}$ & $\begin{array}{l}\text { Bahía de la } \\
\text { Ascensión, en } \\
\text { la Reserva de la } \\
\text { Biósfera de Sian } \\
\text { Ka'an, Quintana Roo }\end{array}$ & & & $\begin{array}{l}\text { Donath- } \\
\text { Hernández, } 1992 \\
\text { **Roccatagliata, } \\
2004\end{array}$ \\
\hline
\end{tabular}

*Son especies que están reportadas para el Pacífico mexicano. **Se refiere a trabajos donde realizaron recopilación de información. ${ }^{ \pm}$Las localidades fueron obtenidas de las coordenadas geográficas. 1 Este estado no se menciona en los artículos originales. 2 Se menciona como Isla de Media en el trabajo original, pero no se especifica la ubicación; posteriormente, en el trabajo de 1992, menciona a Isla de Medina. 3 Se menciona que la localidad es Isla de Enmedio y que queda próximo al puerto de Veracruz, pero esto no se puede corroborar, en la zona norte del estado de Veracruz existe el sistema arrecifal Tuxpan-Lobos en los cuales se encuentran los arrecifes Medio y Enmedio y en la zona centro se encuentra el Parque Nacional Sistema Arrecifal Veracruzano que cuenta con el arrecife Enmedio que también es llamado arrecife Medio.

\section{CONCLUSIÓN}

El estudio de los cumáceos en el Golfo de México y mar Caribe es exiguo, hay estados en los que no existe registro, las localidades han sido pocas y cercanas entre ellas, el trabajo se ha realizado por pocos investigadores y se tienen más de 30 años sin determinar hasta especie los organismos recolectados. Esto muestra la necesidad de un mayor trabajo taxonómico en este grupo para revisar aquellas especies que se indicó serían nuevas, o bien de que se realicen muestreos en localidades no trabajadas.

\section{AGRADECIMIENTOS}

La primera autora agradece al Posgrado en Ciencias Biológicas (Sistemática), UNAM por su apoyo para la realización de este artículo que forma parte de su proyecto de titulación de maestría. Agradecemos especialmente a Jani Jarquín González por su decisivo apoyo con la facilitación de literatura.

\section{LITERATURA CITADA}

Ahyong, Sh. T., J. K. Lowry, M. Alonso, R. N. Bamber, G. A. Boxshall, P. Castro, S. Gerken, G. S. Karaman, J. W. Goy, D. S. Jones, K. Meland, D. Ch. Rogers y J. Svavarsson. 2011. Subphylum Crustacea Brünnich, 1772 (pdf) En: Zhang, Z.-Q. (Ed.) Animal biodiversity: An outline of higher-level classification and survey of taxonomic richness. Zootaxa, 3148: 165-191.

Băcescu, M. 1971. New Cumacea from the littoral waters of Florida (Caribbean Sea). Travaux du Muséum National d'Histoire Naturelle Grigore Antipa, 11: 6-23.

Băcescu, M. 1992. Cumacea II (Fam. Nannastacidae, Diastylidae, Pseudocumatidae, Gynodiastylidae et Ceratucomatidae). En: Gruner, H.-E. y L.B. Holthuis (Eds.), Crustaceorum Catalogue, Vol. 8. Academic Publishing, The Hague, 175-468. 
Băcescu, M. y I. Petrescu. 1999. Ordre des Cumacés. Mémoires de l'Institut océanographique, Monaco, 19: 391-428.

Cházaro-Olvera, S., I. Winfield, M. Ortiz y F. Álvarez. 2002. Peracarid crustaceans from three inlets in the southwestern Gulf of Mexico: new records and range extensions. Zootaxa, 123: $1-16$.

Dexter, D. 1976. The Sandy Beach Fauna of Mexico. The Southwestern Naturalist, 20 (4): 479-485.

Donath-Hernández, F. E. 1987a. Nuevos registros y ampliación de rango geográfico de cuatro especies de cumáceos para el Golfo de California (Crustacea, Cumacea). First record and enlargement of geographic distribution of four species of cumacea for the Gulf of California (Crustacea, Cumacea). Ciencias Marinas, 13 (1): 70-74.

Donath-Hernández, F. E. 1987b. Distribución de los cumáceos (Crustacea, Peracarida) de la Bahía de Todos Santos, Baja California, México. Ciencias Marinas, 13 (4): 35-52.

Donath-Hernández, F. E. 1988a. Cumacea from the Gulf of Mexico and the Caribbean Sea (Crustacea, Peracarida). I: Descriptions of Known Species, New Records and Range Extension. Caribbean Journal of Science, 24 (1-2): 44-51.

Donath-Hernández, F. E. 1988b. Three new species of Cumacea from the Gulf of California (Crustacea, Peracarida). Cahiers de Biologie Marine, 29: 531-543.

Donath-Hernández, F. E. 1992. Cumella (Cumewingia) siankaana n. sp. from the Caribbean coast of Quintana Roo, Mexico (Cumacea Nannastacidae). En: Navarro L. D. y E. Suárez M. 1992. Diversidad Biológica Reserva Biosfera Sian Ka’ an Quintana Roo, México, 2: $163-167$.

Donath-Hernández, F. E. 1993. Cumaceos de Bahía de los Angeles, Baja California, México (Crustacea, Peracarida). Ciencias Marinas, 19 (4): 461-471.

García-Madrigal, M. S., C. Campos-Vázquez y N. E. González. 2002. Sección de crustáceos de la Colección de Referenciade Bentos Costero de ECOSUR. Universidad y Ciencia, 36 (18): 140-148.

Haye, P. A., I. Kornfield y L. Watling. 2004. Molecular insights into Cumacean family relationships (Crustacea, Cumacea). Molecular Phylogenetics and Evolution, 30: 798-809.

Heard, R. W. y D. Roccatagliata. 2009. Cumacea (Crustacea) of the Gulf of Mexico. Pp 1001-1011 En: Felder, D. L. y D. K. Camp (eds), Gulf of Mexico-Origins, Waters and Biota. Biodiversity. Texas A\&M University Press, College Station, Texas.

Hendrickx, M. E. 1995. Checklist of lobster-like decapod crustaceans (Crustacea: Decapoda: Thalassinidea, Astacidea and Palinura) from the eastern tropical Pacific. Anales Inst. Biol., Universidad Nacional Autónoma de México, Serie Zoología, 66: 151-163. 
Hendrickx, M. E., L. García Prieto, B. Yáñez-Rivera, S. Gómez y J. L. Carballo. 2019. Contribución de las instituciones nacionales en el conocimiento de la biodiversidad marina del Pacífico mexicano. Síntesis y proyección hacia el futuro. Geomare Zoológica, 1: 3-50.

Hermoso-Salazar, M. y K. Arvizu-Coyotzi. 2015. Crustáceos del Sistema Arrecifal Veracruzano. Pp: 47-72. En: Granados-Barba, A., L. D. Ortiz Lozano, D. Salas-Monreal y C. González-Gándara (Eds). Aportes al conocimiento del Sistema Arrecifal Veracruzano hacia el Corredor Arrecifal del Suroeste del Golfo de México. Universidad Autónoma de Campeche, 366 pp.

Jarquín-González J. y M. del S. García-Madrigal. 2013. Annotated checklist and keys for cumaceans (Crustacea: Peracarida) from the Tropical Eastern Pacific, with six new species from the Southern Mexican Pacific. Zootaxa, (3): 201-257.

Markham, J. C., F. E. Donath-Hernández, J. L. Villalobos-Hiriart y A. C. Díaz-Barriga. 1990. Notes on the shallow-water marine Crustacea of the Caribbean coast of Quintana Roo, Mexico. Anales del Instituto de Biología de la Universidad Nacional Autónoma de México, Serie Zoología, 61 (3): 405-446.

May, R. M. 1992. How many species inhabit the earth? Scientific American, 267 (4): 42-49.

Muradian-Ciamician, Z. 1980. On some species belonging to the genus Campylaspis (Cumacea, Nannastacidae) from the collections of the Natural History Museum "Grigore Antipa". 21: 73-88.

Ortiz, M., A. Martín y Y. L. Díaz. 2001. Lista y referencias de los crustáceos anfípodos (Amphipoda: Gammaridea) del Atlántico occidental tropical. Revista Biología Tropical, 55 (2): 479-498.

Petrescu, I. 2018. On the Family Nannastacidae (Crustacea, Cumacea) from the Australian Museum Collection. Records of the Australian Museum, 70 (1): 1-111.

Rhada Devi, A. y C. V. Kurian. 1981. Three new species of Cumacea from the Gulf of Mexico. Bulletin of the Department of Marine Sciences, University of Cochin. XII (II): 53-64.

Roccatagliata, D. 2004. Cumacea. En: Llorente, B. J. E., J. J. Morrone, O. P. Yáñez y F. I. Vargas (Eds.) 2004. Biodiversidad, Taxonomía y Biogeografía de Artrópodos de México: Hacia una síntesis de su conocimiento. Facultad de Ciencias UNAM, CONABIO, (IV): 471-481.

Spears, T., R. W. DeBry, L. G. Abele y K. Chodyla. 2005. Peracarid monophyly and interordinal phylogeny inferred from nuclear small-subunit ribosomal DNA sequences (Crustacea: Malacostraca: Peracarida). Proceedings of the Biological Society of Washington, 118 (1): 117-157.

Watling, L. y S. Gerken. 2019. World Cumacea Database. Disponible en: www.marinespecies. org/cumacea (accesado: agosto, 2019).

Winfield, A. I. C. y M. Ortiz. 2011. Crustáceos con bolsa incubadora, (Crustacea: Malacostraca: Peracarida) en La Biodiversidad en Veracruz, estudio de estado, (III): 277-286. 
WoRMS Editorial Board. 2019. World Register of Marine Species. Disponible en: www.marinespecies.org (accesado: agosto, 2019).

Zimmer, C. 1943. Cumaceen des Stillen Ozeans. Archiv für Naturgeschichte, 12 (1): 130-174.

[Recibido: 21 de noviembre, 2019. Aceptado para publicación: 21 de mayo, 2020] 